\section{Commentary: Local chemotherapy with nanoparticles: A ray of light in the dark?}

\author{
Shota Nakamura, MD, and \\ Toyofumi F. Chen-Yoshikawa, MD
}

Malignant pleural mesothelioma is a devastating disease that remains a challenge to treat, necessitating the use of multimodality therapies consisting of chemotherapy, surgery, and radiation therapy. Despite aggressive modes of such treatment, prognosis of the disease remains miserable, and its improvement is one of the major challenges in thoracic oncology. ${ }^{1,2}$ Recently, immune checkpoint inhibitors have also been introduced as a new treatment option for advanced or recurrent malignant pleural mesothelioma. Several strategies are being investigated, and their target is the intrathoracic cavity for local disease control. ${ }^{3,4}$

In this issue of The Journal, Chu and colleagues ${ }^{4}$ reported improved survival in a translational orthotopic murine model of malignant pleural mesothelioma with a nanoparticle-based strategy for intracavitary delivery of chemotherapy to the tumor. Their study was based on the hypothesis that conventional chemotherapy for malignant pleural mesothelioma has been ineffective due to inadequate drug accumulation at tumor sites. Given the clinical benefit reported with early postoperative and long-term intraperitoneal paclitaxel for peritoneal mesothelioma, they performed the initial proof-of-concept study for intrathoracic chemotherapy delivery to pleural mesothelioma using a unique formulation of paclitaxelloaded expansile nanoparticles. They showed that these pH-triggered expansile nanoparticles loaded with paclitaxel preferentially accumulated in tumors and resulted in sustained drug release over time, thereby enhancing drug action and yielding improved survival outcomes.

\footnotetext{
From the Department of Thoracic Surgery, Nagoya University Graduate School of Medicine, Nagoya, Japan.

Disclosures: Authors have nothing to disclose with regard to commercial support.

Received for publication Dec 25, 2019; accepted for publication Dec 26, 2019; available ahead of print Jan 11, 2020.

Address for reprints: Toyofumi F. Chen-Yoshikawa, MD, Department of Thoracic Surgery, Nagoya University Graduate School of Medicine, 65 Tsurumai-cho, Showa-ku, Nagoya 466-8550, Japan (E-mail: tyoshikawa@med.nagoya-u.ac.jp). J Thorac Cardiovasc Surg 2020;160:e169 $0022-5223 / \$ 36.00$

Copyright (c) 2020 by The American Association for Thoracic Surgery https://doi.org/10.1016/j.jtcvs.2019.12.089
}

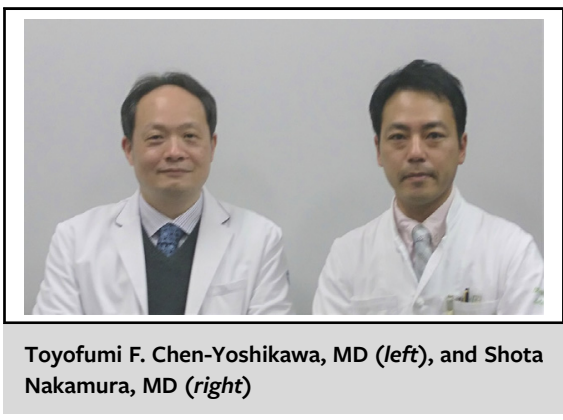

CENTRAL MESSAGE

A novel therapeutic method us-

ing intrathoracic injection of paclitaxel-loaded expansile nanoparticles is promising based on its experimental model mimicking the clinical scenario of mesothelioma.

Chu and colleagues ${ }^{4}$ had reported that expansile nanoparticles could rapidly accumulate in malignant cells, resulting in prolonged drug delivery with high local drug concentrations within the tumor in comparison with those achieved by systemic administration. ${ }^{5,6}$ In the study reported in this issue of The Journal, they demonstrated the same phenomenon in an in vivo setting.

Several questions can be posed for future studies. As Chu and colleagues mentioned in their discussion, paclitaxel is not a typical first-line chemotherapeutic regimen for malignant pleural mesothelioma. In a clinical setting, chemotherapeutic regimens, such as platinum combined with pemetrexed, are usually used as standard therapy for patients with advanced-stage malignant pleural mesothelioma. As the current expansile nanoparticle construct is best suited for encapsulating drugs that are hydrophobic, it is understandable that their expansile nanoparticle was used to encapsulate paclitaxel. However, in terms of current clinical practice, additional nanoparticle constructs are urgently needed to encapsulate drugs with more hydrophilic properties, such as pemetrexed, gemcitabine, and platinum-based drugs. Furthermore, the authors selected only one unique mesothelioma cell line, MSTO-211H, and their results were derived only from this aggressive biphasic cell line. Some clinicians would not proceed to surgical therapy with a biphasic mesothelioma. Hence, the findings of this study would be more reliable if the authors could show similar results 
using other cell lines such as a monophasic epithelioid mesothelioma cell line.

Chu and colleagues are to be congratulated on their excellent basic research leading to translational research. Their results highlight the promise of their strategy as an optimal treatment option for multimodality therapy in patients with malignant pleural mesothelioma.

\section{References}

1. Rusch VW, Giroux D, Kennedy C, Ruffini E, Cangir AK, Rice D, et al. Initial analysis of the international association for the study of lung cancer mesothelioma database. J Thorac Oncol. 2012:7:1631-9.

2. Krug LM, Pass HI, Rusch VW, Kindler HL, Sugarbaker DJ, Rosenzweig KE, et al. Multicenter phase II trial of neoadjuvant pemetrexed plus cisplatin followed by extrapleural pneumonectomy and radiation for malignant pleural mesothelioma. J Clin Oncol. 2009;27:3007-13.

3. Opitz I, Lauk O, Meerang M, Jetter A, Aeschlimann B, Seifert B, et al. Intracavitary cisplatin-fibrin chemotherapy after surgery for malignant pleural mesothelioma: a phase I trial. J Thorac Cardiovasc Surg. 2020;159:330-40.e4.

4. Chu NQ, Liu R, Colby A, Forcrand C, Padera RF, Grinstaff MW, et al. Paclitaxel-loaded expansile nanoparticles improve following cytoreductive surgery in pleural mesothelioma xenografts. J Thorac Cardiovasc Surg. 2020; 160:e159-68.

5. Liu R, Colby AH, Gilmore D, Schulz M, Zeng J, Padera RF, et al. Nanoparticle tumor localization, disruption of autophagosomal trafficking, and prolong drug delivery improve survival in peritoneal mesothelioma. Biomaterials. 2016;102: 175-86.

6. Lei H, Hofferberth SC, Liu R, Colby A, Tevis KM, Catalano P, et al. Paclitaxel-loaded expansile nanoparticles enhance chemotherapeutic drug delivery in mesothelioma 3-dimensional multicellular spheroids. J Thorac Cardiovasc Surg. 2015;149:1417-24.
See Article page e159.

\section{Commentary: One nano-step for murinekind, one giant leap for mesothelioma}

Jordan S. Dutcher, BS, and Jonathan D'Cunha, MD, $\mathrm{PhD}$

Malignant pleural mesothelioma (MPM) is an aggressive neoplasm, in part due to the latent period between onset and presentation, allowing for insidious progression of tumor burden. As treatments have become increasingly aggressive, one would hope that the survival rates for this notoriously lethal variant would improve. Unfortunately, due to limitations of conventional systemic administration of drugs, and their toxic off-target effects, that has not necessarily been the case. The fear of unwanted effects, without significant hope for improved survival, has contributed to a discrepancy in compliance with national mesothelioma treatment guidelines. A retrospective study from the

From the Department of Cardiothoracic Surgery, Mayo Clinic Arizona, Phoenix, Ariz.

Disclosures: Authors have nothing to disclose with regard to commercial support.

Received for publication Dec 28, 2019; accepted for publication Dec 30, 2019; available ahead of print Jan 11, 2020.

Address for reprints: Jonathan D'Cunha, MD, PhD, Department of Cardiothoracic Surgery, Mayo Clinic Arizona, 5777 E Mayo Blvd, Phoenix, AZ 85054 (E-mail: DCunha.Jonathan@mayo.edu).

J Thorac Cardiovasc Surg 2020;160:e170-1

$0022-5223 / \$ 36.00$

Copyright (c) 2020 by The American Association for Thoracic Surgery

https://doi.org/10.1016/j.jtcvs.2019.12.092

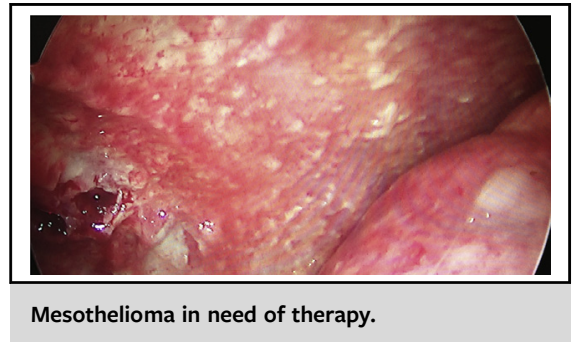

CENTRAL MESSAGE

We review a well-executed study evaluating the increased efficacy and improved survival of nanoparticle-targeted drug delivery in a murine mesothelioma model.

National Cancer Database identified that $31.1 \%$ of patients with mesothelioma did not receive any treatment. ${ }^{1}$ Although the outlined treatment plans are associated with overall survival improvement, these treatments could be improved by implementing a treatment plan that limits the toxicities and potentially improves the oncologic response.

The current landscape of treatment in MPM involves a multimodal approach that includes surgical debulking followed by adjuvant chemotherapy (with or without radiation) for additional disease control. Despite these measures, there is still significant morbidity/mortality 